EPJ Web of Conferences 41, 12010 (2013)

DOI: $10.1051 /$ epjconf/20134112010

C) Owned by the authors, published by EDP Sciences, 2013

\title{
Tunable ultrafast nonlinear optofluidic coupler
}

\author{
Marius Vieweg, ${ }^{1}$ Sebastian Pricking, ${ }^{1}$ Timo Gissibl, ${ }^{1}$ Yaroslav V. Kartashov, ${ }^{2}$ \\ Lluis Torner, ${ }^{2}$ and Harald Giessen ${ }^{1}$ \\ ${ }^{1} 4^{\text {th }}$ Physics Institute and Research Center SCoPE, University of Stuttgart, Pfaffenwaldring 57, 70569 Stuttgart, Germany \\ ${ }^{2}$ ICFO - Institut de Ciencies Fotoniques, and Universitat Politecnica de Catalunya, Mediterranean Technology Park, 08860 \\ Castelldefels, (Barcelona), Spain
}

\begin{abstract}
We present an ultrafast nonlinear coupler fabricated by selectively filling of two strands of a PCF with the liquid $\mathrm{CCl}_{4}$ which exhibits a large Kerr nonlinearity. We demonstrate power dependent switching in this optofluidic device.
\end{abstract}

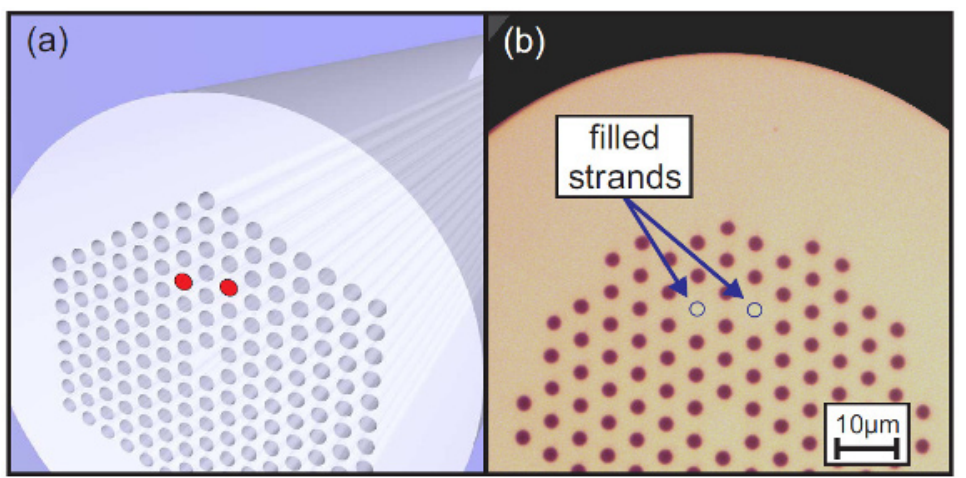

Fig. 1 (a) Illustration of an optofluidic coupler scaffolded by a photonic crystal fiber. (b) Microscope image of a fluid-filled photonic crystal fiber with two $\mathrm{CCl} 4$ strands acting as nonlinear coupler.

In 1982 Jensen [1] proposed an all-optical switch using the material Kerr nonlinearity to control the switching behavior. It is well known that two waveguides placed in close proximity to each other couple together. This results in a sinusoidal back and forth switching of the light between both waveguides in the linear case. The spatial frequency of this process is determined by the overlap of modal fields of the two waveguides. The ratio of output powers is given by the length of the coupling device. The linear coupler device is very common for building fiber based beam splitters.

In the linear regime we cannot control the output power ratio for a fixed length of the device. The situation is changed for the nonlinear coupler (NLC). Here the output becomes dependent on the incoupled intensity and can be tuned to different output power ratios for a fixed length. A variety of realizations in quartz fiber coupler have been presented [2]. They all show the nonlinear dependence of the output ratio. For fused silica fiber couplers as well as for twin-core photonic crystal fibers, the nonlinear coupling is limited by the nonlinear refractive index $n_{2}$ of the host material.

Since it is possible to fill photonic crystal fibers (PCF) selectively [3, 4] with highly nonlinear liquids such as $\mathrm{CCl}_{4}$, two liquid filled strands next to each other can act as NLC device [5] (see 
Fig. 1a). In addition to material properties, the coupling strength $C$ is then defined by the geometry of the photonic structure of the PCF which serves as scaffold for the two liquid cores. A microscope image of a fluid-filled PCF with $\mathrm{CCl}_{4}$ in two strands is shown in Fig. $1 \mathrm{~b} . \mathrm{CCl}_{4}$ has a nonlinear refractive index of about $\mathrm{n}_{2}=15 \cdot 10^{-20} \mathrm{~m}^{2} \mathrm{~W}^{-1}$, which is more than five times higher than that of fused silica, and is nearly transparent around a wavelength of $1.04 \mu \mathrm{m}$.

As mentioned before, we are utilizing the ultrafast Kerr nonlinearity due to molecular reorientation with response times of subpicoseconds [6]. The influence of the delayed nonlinear response on the switching behavior is investigated in [6], where it is also shown that especially for $\mathrm{CCl}_{4}$ these effects are small. Thus the material response is treated in the following as instantaneous.

The liquid cores have a diameter of $2.7 \mu \mathrm{m}$ equal to the hole diameter of the fiber. The PCF has a hole-to-hole distance of $5.6 \mu \mathrm{m}$. With the linear refractive index of $\mathrm{CCl}_{4}$ at $1040 \mathrm{~nm}\left(\mathrm{n}_{0}=1.4503\right)$ just above the index of the fused silica host material, next neighboring holes would give too strong coupling. We decided therefore to fill two holes which are separated by another line of holes. Thus the infiltrated liquid cores are $9.6 \mu \mathrm{m}$ apart from each other (see Fig. 1b), resulting in a coupling length of of about $9 \mathrm{~mm}$. With this geometry we operate in the normal dispersion regime and no temporal soliton formation will occur.

As laser source we use an Yb:Glass oscillator at a wavelength of $1040 \mathrm{~nm}$, a repetition rate of $20 \mathrm{MHz}$, and a pulse duration of $180 \mathrm{fs}$. The laser light is coupled into the liquid core S1 focused with a microscope objective. The output was recorded by a CCD camera.
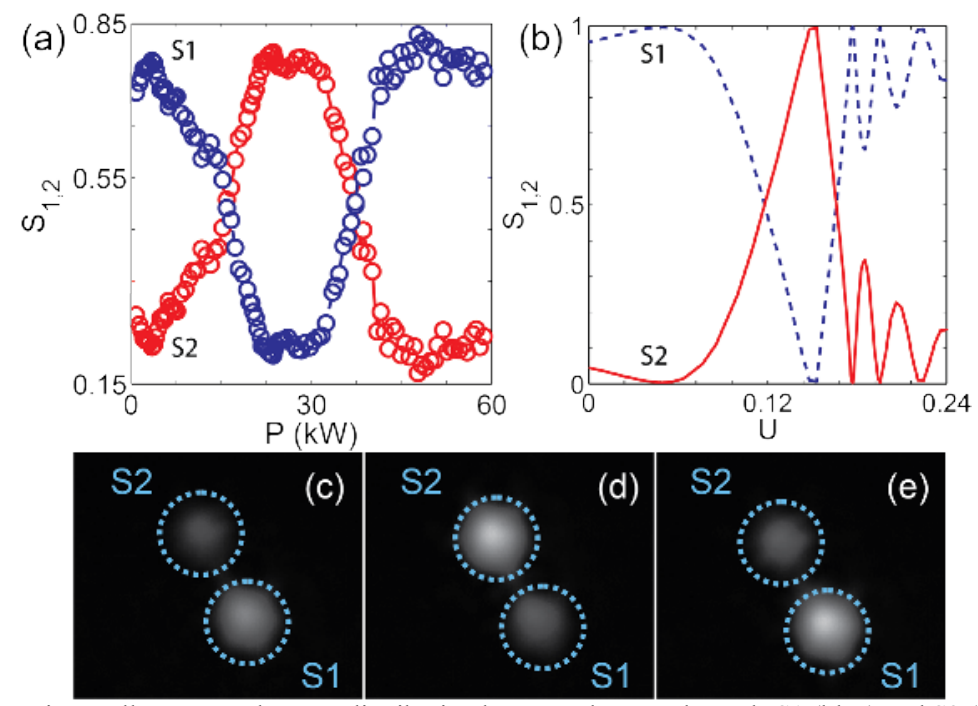

Fig. 2 (a) Experimentally measured power distribution between the two channels S1 (blue) and S2 (red) of the optofluidic coupler versus input power. (b) Theoretically calculated dependencies of power distribution on dimensionless input power U.(c)-(e) Measured intensity distributions at the output of the optofluidic coupler at (c) $3.5 \mathrm{~kW}$, (d) $24.2 \mathrm{~kW}$, and (e) $58.7 \mathrm{~kW}$.

In order to measure the output power ratio a Gauss-function is fitted to the mode image and the ratio of the amplitudes has been extracted from this fit. In Fig. 2a the result for the NLC is shown for room temperature. In this measurement the fiber has a length of $3.6 \mathrm{~cm}$. The measurement [5] shows clearly a power dependent behavior of the output. Fig. 2c-e corresponds to the measured intensity distributions for the three power values of $3.5 \mathrm{~kW}, 24.2 \mathrm{~kW}$, and $58.7 \mathrm{~kW}$, respectively. The region of nonlinear decoupling, for which the induced phase mismatch suppresses further coupling, is for this configuration above the so-called critical power [1] of about $42 \mathrm{~kW}$.

Fig. $2 \mathrm{~b}$ shows an $a b$ initio calculation of the presented double-strand fluid-filled NLC. The solved equation is given by 


$$
i \frac{\partial q}{\partial \xi}=-\frac{1}{2}\left(\frac{\partial^{2} q}{\partial \eta^{2}}+\frac{\partial^{2} q}{\partial \zeta^{2}}\right)-\sigma(\eta, \zeta) q|q|^{2}-R(\eta, \zeta) q
$$

with $q$ being the normalized propagation constant, $\eta$ and $\zeta$ being the normalized transverse coordinates. $\sigma$ de-scribes the normalized spatial nonlinear coefficient and $R$ is the normalized linear refractive index of the fiber device. The numerical solution of this equation gives the dimensionless field amplitude $q$ at the device output. The dimensionless input power $U$ is connected to the input peak power by

$$
P_{0}=\frac{U \lambda^{2}}{4 \pi n_{0} n_{2}}
$$

The agreement between the dynamics of the experiment and the ab initio simulation is very good. Also the approximate model by Jensen [1] (not plotted here) reproduces the results accurately.

In conclusion we have shown a nonlinear application of a selectively fluid-filled PCF. We have built an optofluidic ultrafast nonlinear coupler consisting of two liquid strands embedded in a PCF. Due to the high nonlinearity of the used liquid $\mathrm{CCl}_{4}$, nonlinear switching can be observed in this system and the output can be controlled by the incoupled light. The underlying process for this nonlinearity is the ultrafast Kerr-effect due to molecular reorientation. Therefore this device can be used as ultrafast all-optical switch with switching times below $1 \mathrm{ps}$ which benefits from the enhanced nonlinear properties of the liquid.

The presented concept can be easily extended to more complex waveguide arrays arranged linearly [7] as well as in a 2D structure to observe spatial and temporal soliton effects in these highly nonlinear optofluidic discrete devices. Using our concept, the observation of purely nonlinear lattices should become possible [8].

Acknowledgement. We acknowledge funding by DFG, BMBF, and BW-Stiftung.

1. S.M. Jensen, “The nonlinear coherent coupler," IEEE J. Quant. Electron. 18, 1580-1583 (1982).

2. S.R. Friberg, Y. Silberberg, M.K. Oliver, M.J. Andrejco, M.A. Saifi, and P.W. Smith, "Ultrafast all-optical switching in a dual-core fiber nonlinear coupler," Appl. Phys. Lett. 51, 1135-1137 (1987).

3. M. Vieweg, T. Gissibl, S. Pricking, B.T. Kuhlmey, D.C. Wu, B.J. Eggleton, and H. Giessen, "Ultrafast nonlinear optofluidics in selectively liquid-filled photonic crystal fibers," Opt. Express 18, 25232-25240 (2010).

4. T. Gissibl, M. Vieweg, M.M. Vogel, M. Abdou-Ahmed, T. Graf, and H. Giessen, "Preparation and characterization of a large mode area liquid-filled photonic crystal fiber: transition from isolated to coupled spatial modes," Appl. Phys. B 106, 521 (2012).

5. M. Vieweg, S. Pricking, T. Gissibl, Y. Kartashov, L. Torner, and H. Giessen, "Tunable ultrafast nonlinear optofluidic coupler," Opt. Lett. 37, 1058 (2012).

6. S. Pricking, M. Vieweg, and H. Giessen, "Influence of the retarded response on an ultrafast nonlinear optofluidic fiber coupler," Opt. Express 19, 21673-21679 (2011).

7. M. Vieweg, T. Gissibl, Y. V. Kartashov, L. Torner, and H. Giessen, "Spatial solitons in optofluidic waveguide arrays with focusing ultrafast Kerr nonlinearity," Opt. Lett. 37, 2454 (2012).

8. Y. V. Kartashov, B. Malomed, L. Torner, "Solitons in nonlinear lattices," Rev. Mod. Phys. 83, 247 (2011). 